\title{
Highly efficient laser action in femtosecond-written Nd:yttrium aluminum garnet ceramic waveguides
}

\author{
G. A. Torchia, ${ }^{1, a)}$ A. Rodenas, ${ }^{2}$ A. Benayas, ${ }^{2}$ E. Cantelar, ${ }^{2}$ L. Roso, ${ }^{1}$ and D. Jaque ${ }^{2, b)}$ \\ ${ }^{1}$ Grupo de Óptica, Departamento de Física Aplicada, Facultad de Ciencias Físicas, \\ Universidad de Salamanca, Salamanca 37008, Spain \\ ${ }^{2}$ Departamento de Física de Materiales C-IV, Facultad de Ciencias, Universidad Autónoma de Madrid, \\ 28049 Madrid, Spain
}

(Received 5 December 2007; accepted 11 February 2008; published online 17 March 2008)

\begin{abstract}
We report continuous wave $1.06 \mu \mathrm{m}$ laser operation in an optical waveguide fabricated in a Nd:YAG ceramic by femtosecond laser writing. Single mode and stable laser oscillation have been achieved by using the natural Fresnel reflection for optical feedback. Output laser power in excess of $80 \mathrm{~mW}$ and a laser slope efficiency of $60 \%$ have been demonstrated. (c) 2008 American Institute of Physics. [DOI: 10.1063/1.2890073]
\end{abstract}

Femtosecond direct laser writing (DLW) of transparent materials is attracting much attention because of the unique possibility of three-dimensionally modifying, at the micrometric and submicrometric scale, the optical properties of the irradiated media. This technique has been already proved to be a powerful and flexible tool for the fabrication of a great variety of optoelectronic components such as photonic crystals, diffraction gratings, and optical memories. ${ }^{1-3}$ When femtosecond pulses are focused inside a dielectric material, a permanent change in the refractive index is produced, in such a way that optical waveguides could be generated. This possibility has been already demonstrated in a great variety of glasses and crystals. ${ }^{4-6}$ The further use of the DLW technique for the fabrication of low-loss channel waveguides in laser materials could be highly advantageous with respect to other fabrication approaches, and could also lead to technology breakthroughs in the development of threedimensionally integrated optical circuits.

Among the different solid state laser media, neodymium doped yttrium aluminum garnet (YAG) transparent ceramics are nowadays attracting a great interest because of its advantages over the traditionally used Nd:YAG crystals. These advantages are the lower manufacturing costs, the possibility of high neodymium contents without any decrease in the optical quality of the gain medium, and also the possibility of direct composite fabrication. ${ }^{7}$ As a matter of fact, the laser performance of Nd:YAG ceramics has been found to be equal or even superior to that corresponding to Nd:YAG crystals. ${ }^{8}$ Recently, authors reported on the fabrication of near surface channel waveguides in Nd:YAG ceramics. ${ }^{9}$ Nevertheless, up to date no attempt has been made, to the best of our knowledge, for the fabrication of buried channel waveguides in Nd:YAG ceramics by femtosecond DLW. The possible application of such waveguides as reliable and integrated laser sources is, therefore, still unexplored.

In this letter, we report on the fabrication of buried channel waveguide lasers in Nd:YAG ceramics by using a two line confinement approach. Light confinement has been achieved between two parallel tracks due to filamentation of the femtosecond laser pulses. The possible influence of the waveguide fabrication process on the spectroscopic proper-

\footnotetext{
${ }^{a}$ Also at the Centro de Investigaciones Opticas CIC-Conicet CC124, 1900 La Plata, Argentina.

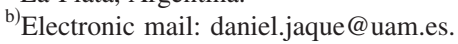

ties of the neodymium ions has been investigated by timeresolved confocal microscopy. We have also demonstrated highly efficient and stable laser oscillation based on the femtosecond written waveguide.

The Nd:YAG ceramic sample used in this work was provided by Baikowski Ltd. (Japan). The sample was a $5 \times 5$ $\times 5 \mathrm{~mm}^{3}$ cube with all its faces polished up to optical quality $(\lambda / 4)$. The nominal $\mathrm{Nd}^{3+}$ concentration was 2 at. \%. The waveguide laser was fabricated by using a Ti:sapphire laser system providing $120 \mathrm{fs}$ pulses at $796 \mathrm{~nm}$ and $1 \mathrm{kHz}$ of repetition rate. The laser beam was focused with a $10 \times$ microscope objective (numerical aperture of 0.3). The translation of the sample was achieved by a $X Y Z$ motorized stage with a spatial resolution of $0.8 \mu \mathrm{m}$. With the linear focus of the objective located $500 \mu \mathrm{m}$ below surface, $29 \mu \mathrm{m}$ long filaments were written with a pulse energy of $11 \mu \mathrm{J}$, which corresponds to a laser power of $85 \mathrm{MW}$, well above the YAG threshold power for self-focusing $(\approx 1 \mathrm{MW})$. Two parallel lines were written separated $20 \mu \mathrm{m}$ by translating the sample with a speed of $50 \mu \mathrm{m} / \mathrm{s}$. Figure 1(a) shows an optical transmission image of the end face. The ability of the resulting structure as an optical waveguide was firstly investigated by end coupling a He-Ne laser $(632.8 \mathrm{~nm})$. Light confinement between the two inscribed channels was observed for both TM and TE polarizations. Moreover, the obtained channel waveguides were found to be stable up to annealing temperatures as high as $1000{ }^{\circ} \mathrm{C}$ without any observable deterioration in its guiding properties.

In order to elucidate the suitability of the obtained optical waveguides as integrated laser sources, we have investigated how the spectroscopic properties of the neodymium ions have been modified by the waveguide fabrication method. Time-resolved microphotoluminescence $(\mu \mathrm{PL})$ experiments were performed. Figure 1(b) shows the room temperature ${ }^{4} F_{3 / 2} \rightarrow{ }^{4} I_{11 / 2} \mu \mathrm{PL}$ spectra obtained at the waveguide region [point $\mathrm{A}$ in Fig. 1(a)], and in an unprocessed area of the Nd:YAG ceramic [point B in Fig. 1(a)]. For these measurements, we used a fiber coupled pulsed diode operating at $808 \mathrm{~nm}$ connected to an Olympus BX41 confocal microscope. As it can be observed, very similar spectra were obtained. The fluorescence lifetime of the ${ }^{4} F_{3 / 2}$ metastable state inside the waveguide was also measured. A fluorescence lifetime of $141 \mu \mathrm{s}$ was found, in good agreement with that obtained at position B (140 $\mu \mathrm{s})$. The invariance of both 

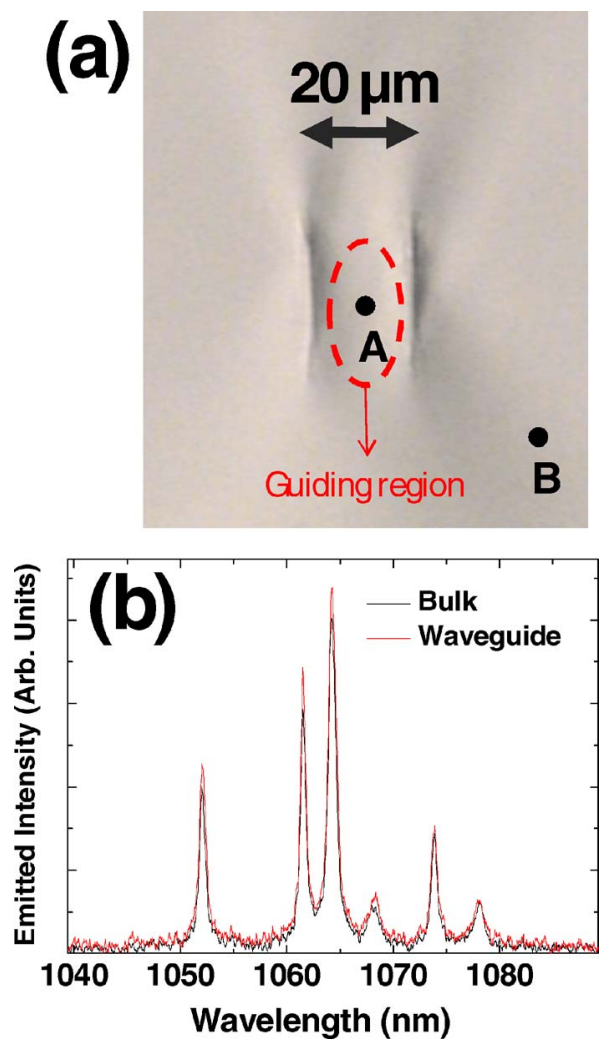

FIG. 1. (Color online) (a) End view of the end face of the waveguide. (b) $\mu \mathrm{PL}$ spectra corresponding to the ${ }^{4} F_{3 / 2} \rightarrow{ }^{4} I_{11 / 2}$ fluorescence channel obtained from the waveguide (point A) and from the bulk material (point B)

fluorescence shape and lifetime inside and out of the waveguide indicate that the spectroscopic properties of the neodymium ions in YAG ceramics have not been deteriorated during the waveguide fabrication procedure; there is not evidence of local disordering and/or presence of optical defects that could eventually act as luminescence killers. This is in accordance with recent works concerning the fabrication of channel waveguides by the two line approach used in this work, ${ }^{10}$ pointing out that the waveguide area corresponds to an almost unmodified region, in such a way that the properties of the original material are preserved. These results reveal that the obtained structure is a good candidate for an integrated waveguide laser since neodymium ions keep their excellent spectroscopic properties in the volume where the waveguide has been produced.

For laser experiments a plane dielectric mirror was attached to the entrance end face. This input mirror was of high $(>80 \%)$ transmittance in the $600-850 \mathrm{~nm}$ range, and of high reflectance $(>99 \%)$ in the $1020-1100 \mathrm{~nm}$ range. No output coupler was attached to the exit end face, what increases the device compactness with respect to other previous approaches. ${ }^{11}$ In these conditions, optical feedback at the exit end face was only provided by the Fresnel reflection. Taking into account the refractive index of Nd:YAG ceramics $\left(n_{0} \approx 1.8\right)$, we have estimated an effective output transmittance and reflectance of $T \approx 92$ and $R \approx 8 \%$, respectively. Optical excitation of the channel waveguide was performed by end-fired coupling a Ti:sapphire continuous wave laser

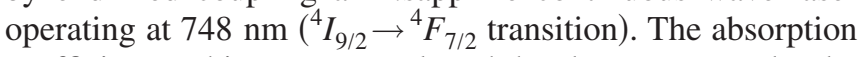
coefficient at this pump wavelength has been measured to be $9.6 \mathrm{~cm}^{-1}$, so that the 5 -mm-long waveguide absorbs virtually all the pump light. The geometry of the experiment was adopted to launch TM propagating modes into the channel

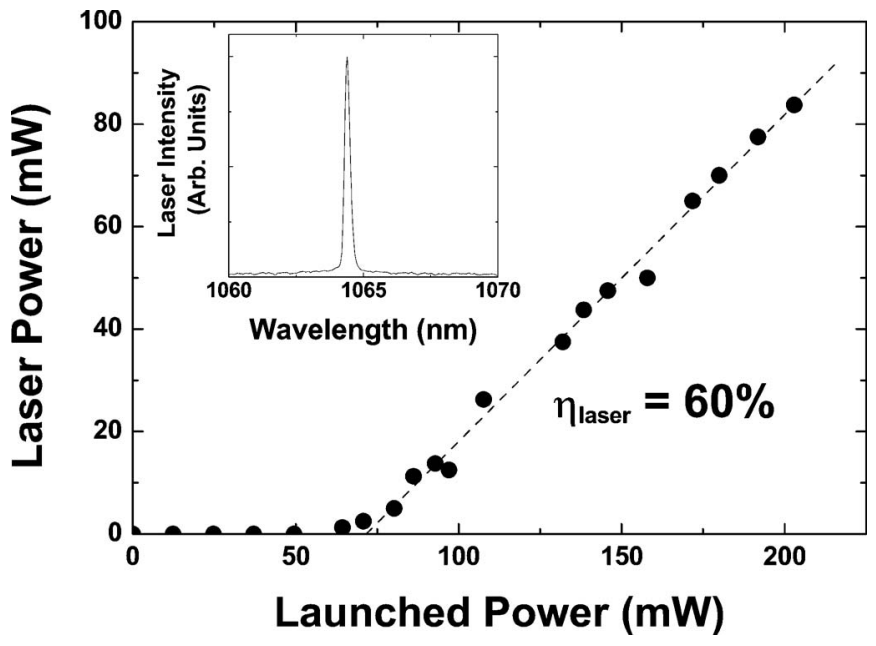

FIG. 2. Laser power as a function of the launched pump power. The inset shows the spectral dependence of the laser radiation.

waveguide. The laser emission from the waveguide was collected from the end face with a second microscope objective. Figure 2 shows the $1.06 \mu \mathrm{m}$ laser power as a function of the launched pump power. For all the pump powers used in this work laser oscillation was found to be TM polarized and single line as can be observed from the inset of Fig. 2. A best fit to the experimental points gives a laser slope efficiency with respect to launched power as high as $60 \%$, and a laser threshold of $68 \mathrm{~mW}$. This laser slope efficiency is among the highest ever reported in a Nd:YAG based waveguide laser. As a matter of fact, it is close to six times the laser slope efficiency previously reported for a femtosecond written waveguide laser fabricated in a Nd:YAG crystal, 1.5 times larger than the laser slope efficiency achieved with an epitaxial grown Nd:YAG waveguide laser and comparable to the laser slope efficiencies reported from Nd:YAG direct bonded waveguides. ${ }^{11-13}$ Assuming a complete absorption of the launched pump power and a 100\% pumping efficiency, the laser slope efficiency $\left(\eta_{\text {laser }}\right)$ can be approximately written as ${ }^{14}$

$$
\eta_{\text {laser }}=\frac{\lambda_{\text {pump }}}{\lambda_{\text {laser }}} \eta_{Q} \frac{\ln \left(\frac{1}{R}\right)}{2 \alpha l+\ln \left(\frac{1}{R}\right)} \frac{d S}{d F},
$$

where $\lambda_{\text {pump }}=748 \mathrm{~nm}$ is the pumping wavelength, $\lambda_{\text {laser }}$ $=1064 \mathrm{~nm}$ is the laser wavelength, $R \approx 0.08$ is the output reflectance (given, in our case, by the Fresnel reflection), $\alpha$ is the loss coefficient, $l=5 \mathrm{~mm}$ is the waveguide length, and $d S / d F$ is the mode-overlap factor. ${ }^{14}$ In our experimental conditions (negligible population in the terminal laser level due to the four level scheme, absence of reabsorption looses and pump beam waist smaller than laser beam waist), it can be assumed that $(d S / d F) \approx 1$. This assumption is in fact consistent with the linear relation between pump and laser powers observed even for pump powers close to threshold. ${ }^{14}$ In expression (1), $\eta_{Q}$ is the pumping efficiency term. There is in the literature certain discrepancy on the exact value of this term in the Nd:YAG system. Different authors have reported values ranging from 0.85 up to $0.95 .^{15,16}$ Taking into account this uncertainty we have assumed in our calculations an intermediate value of 0.9. By substituting the experimental value found for the laser slope efficiency in expression (1), 


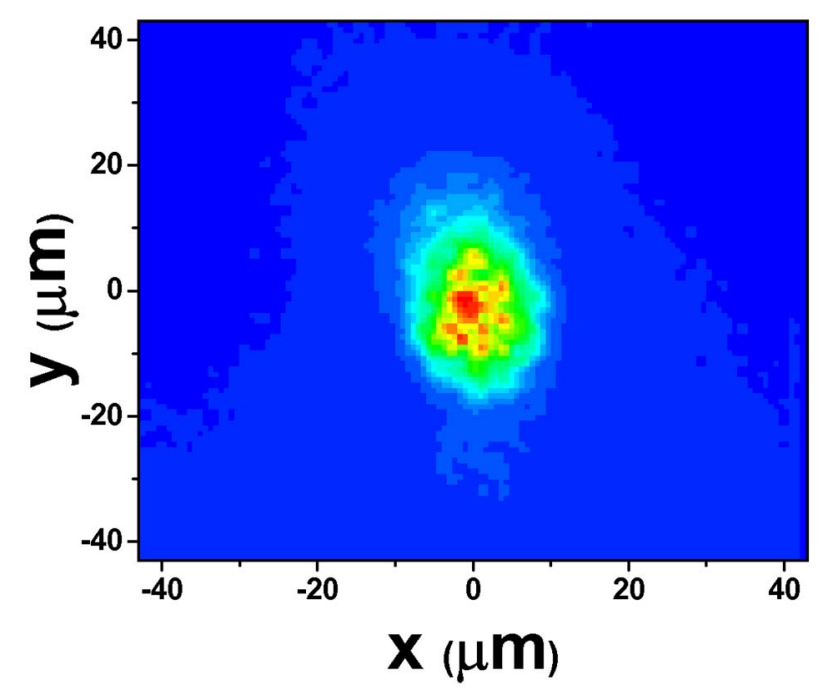

FIG. 3. (Color online) Near field $\mathrm{TM}_{00}$ mode intensity distribution of the laser beam.

we have found $\alpha=0.13 \mathrm{~cm}^{-1}(0.6 \mathrm{~dB} / \mathrm{cm})$. This value has been found to be higher than that reported for a femtosecond written depressed cladding waveguide laser formed in Nd:YAG crystals $(0.3 \mathrm{~dB} / \mathrm{cm}),{ }^{11}$ but comparable to that found in femtosecond laser written surface channel waveguides fabricated in Nd:YAG ceramics $(1 \mathrm{~dB} / \mathrm{cm}) .{ }^{9}$ According to expression (1), the laser slope efficiency of our waveguide laser can be even improved by using longer pump wavelengths, since they would lead to a significant reduction in the quantum defect between pump and laser photons which, in turn, will be accompanied by a reduction in the pump induced thermal loading and, therefore, in the undesirable thermal effects. ${ }^{17}$

The near field $\mathrm{TM}_{00}$ mode intensity distribution was measured with a charge coupled device camera. The measured intensity distribution is shown in Fig. 3. Mode halfwidth sizes, measured at $1 / e^{2}$ intensity level are 11 and $16 \mu \mathrm{m}$ in the transverse and lateral directions, respectively. Based on the obtained near field profiles, we have calculated, in a first order approximation, a refractive index change of $\Delta n \approx 7 \times 10^{-4}$ within the laser waveguide mode. ${ }^{18}$ This value is comparable to that previously reported for similar waveguides fabricated in lithium niobate crystals $(\Delta n \approx 2$ $\times 10^{-4}$ ) (Ref. 10) and also similar to the refractive index change estimated in femtosecond written depressed cladding waveguides fabricated in Nd:YAG crystals $\left(\Delta n \approx 4 \times 10^{-4}\right) .{ }^{11}$ The near field profile of pump mode was also registered. The pump beam spot sizes (measured as the half width to $1 / e^{2}$ intensity) have been found to be 10 and $13 \mu \mathrm{m}$ in the transverse and lateral directions, respectively. The predicted threshold absorbed power is given by ${ }^{14}$

$$
P_{\mathrm{th}}=\frac{\pi h v_{p}\left(w_{L}^{2}+w_{P}^{2}\right)}{4 \sigma_{\mathrm{em}} \tau_{\mathrm{fl}}}\left[2 \alpha l+\ln \left(\frac{1}{R}\right)\right],
$$

where $\sigma_{\mathrm{em}} \approx 3.5 \times 10^{-23} \mathrm{~m}^{2}$ is the stimulated emission cross section, ${ }^{19} v_{p}$ is the pump frequency, $\tau_{\mathrm{fl}}=140 \mu \mathrm{s}$ is the fluorescence lifetime, and $w_{L}$ and $w_{p}$ are the averaged laser and pump beam waists. The laser threshold predicted from ex- pression (2) was $38 \mathrm{~mW}$. Thus, the predicted threshold is lower than the experimentally obtained $(68 \mathrm{~mW})$. Several mechanisms could account for this discrepancy, such as the presence of energy transfer upconversion processes. ${ }^{19}$

In summary, high thermally stable buried channel laser waveguides have been fabricated in Nd:YAG ceramics by the DLW technique. Time-resolved $\mu \mathrm{PL}$ confocal microscopy experiments revealed that the spectroscopic properties of the neodymium ions have not been affected by the waveguide fabrication procedure. By using the Fresnel reflections for optical feedback, laser action at $1064 \mu \mathrm{m}$ has been demonstrated from the obtained waveguides with laser slope efficiencies as high as $60 \%$, and output laser powers in excess of $80 \mathrm{~mW}$. This laser outputs show a very high stability which clearly confirms the excellent mechanical and thermal properties of the Nd:YAG ceramic host besides the excellent optical properties of this matrix, and the versatility of the direct laser writing technique to construct highly efficient miniaturized three-dimensional devices.

This work has been supported by the Spanish Ministerio de Educación y Ciencia (MAT2004-03347, TEC2004-05260C02-02, and MAT2005-05950) by FEDER founds (FIS200501351), by the Universidad Autónoma de Madrid and Comunidad Autonoma de Madrid (projects MICROSERES-CM and CCG06-UAM/MAT-0347), and by the Junta de Castilla y León (Grant No. SA026A05). G.A.T. wishes to thank to the Spanish Ministerio de Educacion y Ciencia (Project No. FIS2006-04151), to the Agencia de Promocion Cientifica y Tecnologica de Argentina (Project No. PICT 15210), and to the Conicet for the financial support received.

${ }^{1}$ G. Zhou and M. Gu, Opt. Lett. 31, 2783 (2006).

${ }^{2}$ K. Kawamura, T. Ogawa, N. Sarukura, M. Hirano, and H. Hosono, Appl. Phys. B: Lasers Opt. 71, 119 (2000).

${ }^{3}$ K. Miura, J. Qiu, S. Fujiwara, S. Sakaguchi, and K. Hirao, Appl. Phys. Lett. 80, 2263 (2002).

${ }^{4}$ M. Hughes, W. Yang, and D. Hewak, Appl. Phys. Lett. 90, 131113 (2007). ${ }^{5}$ G. Della Valle, R. Osellame, N. Chiodo, S. Taccheo, G. Cerullo, P. Laporta, A. Killi, U. Morgner, M. Lederer, and D. Kopf, Opt. Express 13, 5976 (2005).

${ }^{6}$ A. Nejadmalayeri and P. Herman, Opt. Express 15, 10842 (2007).

${ }^{7}$ Y. Sato, A. Ikesue, and T. Taira, IEEE J. Sel. Top. Quantum Electron. 13, 838 (2007).

${ }^{8}$ J. Lu, M. Prabhu, J. Xu, K. Ueda, H. Yagi, T. Yanagitani, and A. A. Kaminskii, Appl. Phys. Lett. 77, 3707 (2000).

${ }^{9}$ G. A. Torchia, P. Meilan, A. Rodenas, D. Jaque, C. Mendez, and L. Roso, Opt. Express 15, 13266 (2007).

${ }^{10}$ J. Thomas, M. Heinrich, J. Burghoff, S. Nolte, A. Ancona, and A. Tünnermann, Appl. Phys. Lett. 91, 151108 (2007).

${ }^{11}$ A. G. Okhrimchuk, A. V. Shestakov, I. Khrushchev, and J. Mitchell, Opt. Lett. 30, 2248 (2005).

${ }^{12}$ I. Chartier, B. Ferrand, D. Pelenc, S. J. Field, D. C. Hanna, A. C. Lage, D. P. Sheperd, and A. C. Tropper, Opt. Lett. 17, 810 (1992).

${ }^{13}$ J. I. Mackenzie, C. Li, and D. P. Shepherd, IEEE J. Quantum Electron. 39, 493 (2003).

${ }^{14}$ W. P. Risk, J. Opt. Soc. Am. B 5, 1412 (1988).

${ }^{15}$ N. P. Barnes and B. M. Walsh, OSA Proc. Adv. Solid-State Lasers 68, 284 (2002).

${ }^{16}$ W. Koechner, Solid State Laser Engineering (Springer, Berlin, 1999).

${ }^{17}$ Z. D. Luo, Y. D. Huang, M. Montes, and D. Jaque, Appl. Phys. Lett. 85, 715 (2004).

${ }^{18}$ A. W. Snyder and J. D. Love, Optical Waveguide Theory (Chapman and Hall, London, 1983).

${ }^{19}$ S. Guy, C. L. Bonner, D. P. Shepherd, D. C. Hanna, and A. C. Tropper, IEEE J. Quantum Electron. 34, 900 (1998). 